\title{
Tunneling Effects in Electromagnetic Wave Scattering by Nonspherical Particles: A Comparison of the Debye Series and Physical-geometric Optics Approximations
}

\author{
Lei Bi and Ping Yang \\ Department of Atmospheric Sciences, Texas A\&M University, College Station, Texas, 77843
}

For Publication in

Special Issue of Electromagnetic and Light Scattering Conference XV in

Journal of Quantitative Spectroscopy and Radiative Transfer

November 2015 
Corresponding author address: Dr. Lei Bi, Department of Atmospheric Sciences, Texas A\&M University, College Station, TX 77843, USA; Email address: bilei@tamu.edu 


\section{Abstract}

The accuracy of the physical-geometric optics (PG-O) approximation is examined for the simulation of electromagnetic scattering by nonspherical dielectric particles. This study seeks a better understanding of the tunneling effect on the phase matrix by employing the invariant imbedding method to rigorously compute the zeroth-order Debye series, from which the tunneling efficiency and the phase matrix corresponding to the diffraction and external reflection are obtained. The tunneling efficiency is shown to be a factor quantifying the relative importance of the tunneling effect over the Fraunhofer diffraction near the forward scattering direction. Due to the tunneling effect, different geometries with the same projected cross section might have different diffraction patterns, which are traditionally assumed to be identical according to the Babinet principle. For particles with a fixed orientation, the PG-O approximation yields the external reflection pattern with reasonable accuracy, but ordinarily fails to predict the locations of peaks and minima in the diffraction pattern. The larger the tunneling efficiency, the worse the PG-O accuracy is at scattering angles less than 90 degrees. If the particles are assumed to be randomly oriented, the PG-O approximation yields the phase matrix close to the rigorous counterpart, primarily due to error cancellations in the orientation-average process. Furthermore, the PG-O approximation based on an electric field volume-integral equation is shown to usually be much more accurate than the Kirchhoff surface integral equation at side-scattering angles, particularly when the modulus of the complex refractive index is close to unity. Finally, tunneling efficiencies are tabulated for representative faceted particles.

Key words: Debye series, Diffraction, Reflection, Tunneling 


\section{Introduction}

Accurate methods that solve macroscopic Maxwell's equations and approximate methods based on physicalgeometric optics (PG-O) principles represent two major classes of numerical methods for computing the optical properties of nonspherical particles [1-3]. PG-O approximation methods are used for nonspherical particles that have sizes much larger than the incident wavelength. On one hand, PG-O approximation methods continue to gain popularity due to their simplicity in implementation, computational efficiency, and reasonable accuracy in computing the optical properties of large nonspherical particles. On the other hand, criteria used to justify the applicability of the PG-O approximation for specific applications remain vague. In particular, for example, "the larger the size parameter, the better the PG-O accuracy" might not be always true because of the involved complexity in relating semiclassical optical mechanisms (e.g., tunneling/edge effects and surface waves $[4,5]$ ) missed in the near-field calculation to their impacts on the optical properties (namely, the extinction and absorption efficiencies, and the phase matrix). The primary reason leading to the aforesaid vagueness is that the PG-O approximations stem fundamentally from physical rationales (featured with a fusion of theoretical foundations and empirical formalism without tuning parameters for better accuracy) rather than on a rigorous solution to Maxwell's equations. Moreover, PG-O methods based on equivalent electromagnetic wave near-to-far-field equations may yield dissimilar results. Therefore, there has been a persistent appeal to better understand the uncertainties and the applicability regimes of the PG-O methods $[6]$.

It is customary to examine PG-O accuracy based on direct comparison of approximate PG-O optical properties [7-15] with their rigorous counterparts computed from numerically exact methods, including the finitedifference time domain (FDTD) and pseudo-spectral time domain (PSTD)[16-19], the discrete dipole approximation (DDA)[20-22], and the T-matrix method [23-27]. From an application perspective, such a comparison is indispensible for quantifying the PG-O accuracy and delineating its applicability regimes. Thanks to successful implementations of numerically exact methods to solve the scattering problem of large particle sizes, the validation of PG-O approximations [28-30] can be carried out in a relatively large size parameter range. Due to the diversity of PG-O approximations, several PG-O algorithms were reviewed in $\mathrm{Bi}$ and Yang [31], including the conventional geometric optics method (CGOM), the improved geometric optics method (IGOM) and the physical-geometric optics hybrid (PGOH) method. The CGOM inaccuracy was assessed in [32] 
together with errors propagating to downstream applications in remote sensing and radiative transfer simulations involving ice clouds. As reported recently by Zhou and Yang [33], the IGOM fails to reproduce a backscattering peak revealed by the T-matrix and PSTD solutions for roughened ice crystals. The backscattering peak associated with the phase function of roughened ice crystals has been shown to play a critical role in retrieving the microphysics of cirrus clouds based on spaceborne lidar, the Cloud Aerosol Lidar with Orthogonal Polarization (CALIOP), on the Cloud Aerosol Lidar and Infrared Pathfinder Satellite Observations (CALIPSO) satellite [33]. Thus, there is a pressing need to understand the physical mechanism leading to enhanced backscattering.

A more suitable approach to studying the semiclassical scattering effects missed in the PG-O approximation is a scattering amplitude expansion technique, pioneered by Debye [34] in 1908, which reformulates the scattering solution from Maxwell's equations as a new series, referred to as the Debye series. Similar to the PG-O approximation, the Debye series decomposes the scattering amplitude as a sum of terms corresponding to diffraction, reflection, and higher-order transmitted light [34,35]; but, unlike the PG-O approximation, the Debye series is rigorously grounded on electromagnetic wave theory. Through the comparison of PG-O results with their counterparts derived from the Debye series, semiclassical scattering effects can be revealed in significant detail as a mix of wave and ray characteristics. For example, in the case of a sphere, the contributions of complex rays and surface waves to the far field are displayed explicitly [36]. The application of complex angular momentum theory to the Debye series of a homogeneous sphere provides a new physical framework to understand the atmospheric rainbow and glory [37]. Lock and Laven [38,39] extended the Debye series of a sphere to the time domain to investigate the diffraction, reflection and surface wave mechanisms involved in the scattering process. However, an extension of Debye's approach to three-dimensional nonspherical particles is much more complicated in terms of both theoretical formalism and stable computational programs. Most available studies are limited to layered spheres and spheroids [37-42], where the method of separation of variables is applicable. Important progress with reference to nonspherical particles with more general shapes was made by $\mathrm{Xu}$ et al [43], who developed a theoretical formalism based on the extended boundary condition method. Alternatively, Bi et al. [44] extended an invariant imbedding approach originally developed for computing the T-matrix [45-49] to compute the zeroth-order Debye series for arbitrarily shaped nonspherical particles. 
The intent of this study is to apply the Debye series technique to assess the PG-O accuracy in conjunction with electromagnetic scattering by nonspherical particles and improve the current level of knowledge of the semiclassical scattering mechanisms. It would be valuable to reveal the conditions that could give rise to significant tunneling effects near the particle edges that have obvious impacts on the optical properties. With an exploratory effort in the case of a nonspherical particle [44], we showed that the tunneling efficiency could be obtained from the zeroth-order Debye series, which contains the Fraunhofer diffraction, reflection and tunneling information. The present study further explores the use of the technique developed in [44] to calculate the phase matrix and analyze thoroughly the PG-O approximations for computing diffraction and direct reflection for a general nonspherical particle. With reference to strongly absorbing particles, the methods such as the T-matrix, the FDTD or the DDA can be employed to study the aforementioned accuracy [50-52] because the higher-order refracted light can be reasonably neglected. We note that the present approach based on the zeroth-order Debye series does not suffer from such limitations on the refractive index. Therefore, the PG-O accuracy for computing diffraction and reflection can be studied for particles with significantly different absorptions.

The remaining part of this paper is organized as follows: Section 2 starts a conceptual review of diffraction, reflection and tunneling. The benefits of separating diffraction and reflection from scattering are discussed. We then outline the invariant imbedding Debye method and the PG-O formulas used for computing the phase matrix corresponding to the diffraction and reflection. In particular, a new analytical algorithm is presented to improve the efficiency and accuracy for computing integrals associated with Wigner d-functions. In Section 3, we present numerical results of rigorous Debye's solutions and PG-O approximations. The PG-O accuracy and tunneling effects are illustrated through various numerical experiments. Finally, the conclusions of this study are given in Section 4.

\section{Diffraction, Reflection and Tunneling}

\subsection{Conceptual Basis}

An extensively used term "diffraction" with different implications exists in the literature [4]. "Diffraction" in most cases describes various wave phenomena associated with wave interference according to the HuygensFresnel principle. The term "rigorous diffraction theory" normally refers to the Sommerfeld perfectly 
conducting half-plane problem [53] or the scattering by a three-dimensional particle (e.g., the Lorenz-Mie theory). According to van de Hulst [4], in the problem of light scattering by a particle, diffraction refers to the asymptotic pattern of the phase function at very small scattering angles and for very large particle sizes. The physical mechanism of diffraction by a three-dimensional particle is associated with diffraction by an aperture in an infinite screen; the aperture is of the same shape and size as the geometric projected area of the particle according to the Babinet principle [4]. Although the aforementioned concept of diffraction is intuitive based on the Huygens principle and a quantitative calculation of diffraction can be obtained from the Kirchhoff formula, unlike extinction and scattering, a theoretical formalism for rigorous calculation of "diffraction" for a general three-dimensional macroscopic particle does not exist. The PG-O methods based on different but equivalent near-to-far-field equations to calculate the diffraction pattern may lead to different results. If exact computational methods are employed for the scattering calculation, diffraction information is inherently contained in the optical properties. Laven [54] analyzed the problem of separating diffraction from scattering as a challenging problem and suggested a partial solution based on the time domain approach [38]. Effort has been undertaken by Lock and Laven [39] to "define" diffraction by a sphere from the Lorenz-Mie theory.

Unlike diffraction, known as a wave phenomenon, reflection is more like a geometrical concept. In the framework of geometric optics, the intensity and polarization state of reflected light from a particle surface are determined from a local application of Snell's law and Fresnel's formula. However, geometric-optics laws are obtained from the incidence of a plane wave upon an infinite plane. The application of geometric optics to determine the reflection is fundamentally questionable near the boundary of a particle. Similar to diffraction, a rigorous definition of reflection by a three-dimensional nonspherical particle does not exist.

Therefore, diffraction and reflection do not have rigorous definitions for a three-dimensional particle from a mathematical point of view. PG-O approximations based on the Kirchhoff surface integration can give a quantitative definition of diffraction as well as reflection but neglect subtle wave-particle interactions near the particle boundary. There are also other semi-empirical theories for more sophisticated treatment of the diffraction and reflection for perfectly conducting particles, such as the geometric theory of diffraction (GTD) [55], and the physical theory of diffraction (PTD) [56]. The hybrid numerical-asymptotic approximation has been suggested to be a promising approach to improve the PG-O approximation near the particle boundary [57]. 
However, a quantity associated with diffraction in conjunction with reflection can be defined without any ambiguity. For example, in the case of a perfectly conducting particle, the scattering solution is due to diffraction plus reflection. For a transparent dielectric particle, diffraction and reflection can be defined through the zeroth-order Debye series. Unlike the PG-O approximation, the sum of diffraction and reflection can be rigorously separated from the scattering solution. Diffraction in conjunction with reflection derived from the zeroth-order Debye series contains much more information than their counterparts defined in the PG-O approximations. Therefore, it is beneficial to separate diffraction and reflection from scattering for a dielectric particle to have a better understanding of the effects associated with the edge domain on the optical properties. For clarity, the term "Fraunhofer diffraction" for a three-dimensional particle is used to indicate the diffraction pattern computed from the PG-O approximations.

First, the tunneling/edge-effect efficiency can be defined. According to the optical theorem, the extinction efficiency can be determined from the scattering amplitude in the forward scattering direction [4],

$$
Q_{e x t}=\frac{2 \pi}{k^{2} A} \operatorname{Re}\left[S_{11}\left(\theta=0^{0}\right)+S_{22}\left(\theta=0^{0}\right)\right]
$$

where $k$ is the wave number defined as $k=2 \pi / \lambda$ in which $\lambda$ is the incident wavelength, $A$ is the projected area, $\operatorname{Re}\left[\right.$ ] indicates the real part of the argument within the brackets, $\theta$ is the scattering angle, and $S_{11}$ and $S_{22}$ are diagonal elements of the amplitude scattering matrix. The extinction mechanism can be understood by resolving the physical processes that contribute to the forward scattering amplitude. If we consider only diffraction and external reflection, the extinction efficiency predicted from the PG-O approximations is 2 . It means that the extinction cross-section arises from the Fraunhofer diffraction and blocking effects. Higher order transmissions change the forward scattering amplitude and phases, causing an interference effect. From the zeroth-order Debye series, the extinction efficiency is larger than 2 and the extra contribution is an edge effect or tunneling effect associated the wave front portion that grazes or nearly grazes the particle's boundary (hereafter, edge effect and tunneling effect are used interchangeably). Table 1 summarizes extinction mechanisms outlined from interpreting the results from spheres and cylinders [4]. Rigorous T-matrix results contain all the physical mechanisms. In the PG-O approximation, the edge effect and surface waves are neglected in accordance with the employed physical rationales. Because the total diffraction and blocking 
efficiency is a constant (i.e., 2), the edge effect can be obtained from the zeroth-order Debye's series. Adding the edge-effect efficiency to the PG-O approximation improves the PG-O extinction efficiency [58-61]. Semiempirical laws obtained to quantify the edge-effect efficiency create new ways to improve the PG-O approximation. For example, the principle of local field has been used to incorporate the edge effect contribution to the extinction efficiency of spheroids [61]).

Second, the zeroth-order Debye series can be employed to examine the PG-O accuracy in computing the diffraction and reflection, analyze the edge effect on the phase matrix, and gain a more insightful understanding of the uncertainties of the PG-O approximations. An increasing body of literature has been devoted to improving the PG-O accuracy in the case of perfectly conducting particles. The difference between the PG-O approximation and rigorous results is practically defined as edge effects, semi-classical effects or fringe-field effects. A correct depiction of these differences in many instances comes with new mathematical tools to improve the PG-O approximations. Several well-known examples are the GTD [55], the PTD [56], and Fock's theory of diffraction in penumbra regions [62]. However, the condition of perfect conduction is an idealized assumption. Analytical asymptotic methods derived from Maxwell's equations for a dielectric particle are unavailable for a nonspherical particle. The zeroth-order Debye series would provide valuable information for imperfectly conducting nonspherical particles, where diffraction and reflection are the only observable quantities.

Third, the zeroth-order Debye series can be applied to compute the diffraction pattern of an aperture in an imperfectly conducting screen of finite thickness. For example, the zeroth-order Debye series can be first applied to compute the diffraction of a disk. The scattering in the forward hemisphere is "equivalent" to the diffraction by the aperture, but the effects of the material and its thickness are taken into account. The influences of finite thickness and finite dielectric constants have received increasing theoretical and experimental attention due to its applications in nano-optics [63].

The higher-order Debye series are also indispensible for interpreting some additional semiclassical physical mechanisms, such as the Goos-Hänchen effect upon total reflection within ice crystals, and the transformation of surface waves to propagating waves. Such phenomena are beyond the scope of the present paper. However, we note that the contribution to the extinction efficiency from the surface waves and the 
semiclassical correction of the interference between diffraction and transmission is relatively small in comparison with the edge effect obtained from the zeroth-order Debye series.

\subsection{Computing the Zeroth-order Debye Series}

Here we review the fundamental equations to compute the zeroth-order Debye series [44] and present a new algorithm for computing integrals associated with the Wigner d-function.

For a general nonspherical particle, to establish the relationship between ingoing spherical waves and outgoing reflected waves, an R-matrix is defined as follows,

$$
\left[\begin{array}{c}
\tilde{p} \\
\tilde{q}
\end{array}\right]=\mathbf{R}\left[\begin{array}{c}
\tilde{a} \\
\tilde{b}
\end{array}\right],
$$

where $(\tilde{a}, \tilde{b})$ and $(\tilde{p}, \tilde{q})$ are coefficients in expanding the incident spherical wave and the associated reflected wave with respect to appropriate vector spherical wave functions,

$$
\begin{aligned}
& \mathbf{E}^{i n c}(\mathbf{r})=\sum_{l=1}^{\infty} a_{l} \tilde{\mathbf{M}}_{l}(k r)+b_{l} \tilde{\mathbf{N}}_{l}(k r), \\
& \mathbf{E}^{r e f}(\mathbf{r})=\sum_{l=1}^{\infty} \tilde{p}_{l} \mathbf{M}_{l}(k r)+\tilde{q}_{l} \mathbf{N}_{l}(k r) .
\end{aligned}
$$

In Eqs. (3) and (4), $\tilde{\mathbf{M}}$ and $\tilde{\mathbf{N}}$ are vector spherical wave functions constructed from spherical Hankel functions of the second kind, and $\mathbf{M}$ and $\mathbf{N}$ are similar to $\tilde{\mathbf{M}}$ and $\tilde{\mathbf{N}}$ except that spherical Hankel functions of the second kind are replaced with the first kind. Note that $l=n(n+1)+m$ is an index defined to combine the angular momentum number $n$ and the projected angular momentum number $m$. Inversely, $n(l)=\operatorname{ceil}(\sqrt{l+1})-1$, where ceil is a function to round a variable to the nearest integer greater than or equal to itself, and 
$m(l)=l-n(n+1)$. In the following equations, $l^{\prime}, n^{\prime}$, and $m^{\prime}$ are indices defined in the same way as $l, n$, and $m$.

To compute the phase matrix, the zeroth-order T-matrix is defined as

$$
\mathbf{T}^{0}=-\frac{1}{2}(\mathbf{1}-\mathbf{R})
$$

$\mathbf{T}^{0}$ has the same symmetry relationships as the T-matrix $\mathbf{T}[1]$. For strongly absorptive particles, it can be shown that $\mathbf{T} \approx \mathbf{T}^{0}$. In Bi et al. [44], we have derived an invariant imbedding equation of the $\mathbf{R}$ matrix similar to the T-matrix; however, the equation is only applicable to quasi-spherical particles due to numerical convergence problems. In practical numerical computations, we solve the following matrix Riccati differential equation,

$$
\frac{d \mathbf{T}^{0}(k r)}{d(k r)}=i\left\{\operatorname{Re}\left[\mathbf{H}^{T}(k r)\right]+\mathbf{T}^{0}(k r) \mathbf{H}(k r)^{T}\right\} \mathbf{U}(k r)\left\{\operatorname{Re}[\mathbf{H}(k r)]+\mathbf{H}(k r) \mathbf{T}^{0}(k r)\right\}
$$

where $\mathbf{T}^{0}(\mathrm{kr})$ indicates the zeroth-order T-matrix in conjunction with a partial volume of the particle within a sphere of radius $k r$, and the other quantities are defined by

$$
\begin{gathered}
H_{l l^{\prime}}=\delta_{l l^{\prime}}\left[\begin{array}{cc}
h_{n}^{(1)}(k r) & 0 \\
0 & \frac{1}{k r} \frac{\partial}{\partial r}\left[r h_{n}^{(1)}(k r)\right] \\
0 & \sqrt{n(n+1)} h_{n}^{(1)}(k r) / k r
\end{array}\right] \\
U_{l l^{\prime}}=f_{l l^{\prime}} \int_{0}^{2 \pi} d \phi \int_{0}^{\pi} d \theta \sin \theta e^{-i\left(m-m^{\prime}\right) \phi}\left[\tilde{m}^{2}(r, \theta, \phi)-1\right]\left[\begin{array}{ccc}
U^{1} & -U^{2} & 0 \\
U^{2} & U^{1} & 0 \\
0 & 0 & U^{3}
\end{array}\right], \\
f_{l l^{\prime}}=k^{2} r^{2}(-1)^{m+m^{\prime}}\left[\frac{2 n+1}{4 \pi n(n+1)}\right]^{1 / 2}\left[\frac{2 n^{\prime}+1}{4 \pi n^{\prime}\left(n^{\prime}+1\right)}\right]^{1 / 2} \\
U^{1}=\pi_{m n}(\theta) \pi_{m^{\prime} n^{\prime}}(\theta)+\tau_{m n}(\theta) \tau_{m^{\prime} n^{\prime}}(\theta),
\end{gathered}
$$




$$
\begin{gathered}
U^{2}=i\left[\pi_{m n}(\theta) \tau_{m^{\prime} n^{\prime}}(\theta)+\tau_{m n}(\theta) \pi_{m^{\prime} n^{\prime}}(\theta)\right], \\
U^{3}=\sqrt{n(n+1)} \sqrt{n^{\prime}\left(n^{\prime}+1\right)} d_{0 m}^{n}(\theta) d_{0 m^{\prime}}^{n^{\prime}}(\theta),
\end{gathered}
$$

where $\tilde{m}$ is the refractive index, $h_{n}^{(1)}(k r)$ is a spherical Hankel function of the first kind, $d_{0 m}^{n}$ is the Wigner d-function and $\pi_{m n}(\theta)=m / \sin \theta d_{0 m}^{n}(\theta)$ and $\tau_{m n}(\theta)=d / d \theta\left[d_{0 m}^{n}(\theta)\right]$.

Practically, to compute the zeroth-order Debye series from Eq. (5), we select the initial value $\mathbf{T}^{0}$ at the radius of an inscribed sphere, given by

$$
\mathbf{T}_{l l^{\prime}}^{0}=\delta_{l l^{\prime}}\left[\begin{array}{cc}
-b_{n(l)}^{0} & 0 \\
0 & -a_{n(l)}^{0}
\end{array}\right]
$$

where

$$
\begin{aligned}
& b_{n(l)}^{0}=\frac{-\left(x j_{n}(x)\right)^{\prime} \varsigma_{n}^{(2)}(\tilde{m} x)+\tilde{m}\left(x j_{n}(x)\right) \varsigma_{n}^{(2)^{\prime}}(\tilde{m} x)}{-\varsigma_{n}^{(1)^{\prime}}(x) \varsigma_{n}^{(2)}(\tilde{m} x)+\tilde{m} \varsigma_{n}^{(1)}(x) \varsigma_{n}^{(2)^{\prime}}(\tilde{m} x)}, \\
& a_{n(l)}^{0}=\frac{-\tilde{m}\left(x j_{n}(x)\right)^{\prime} \varsigma_{n}^{(2)}(\tilde{m} x)+x j_{n}(x) \varsigma_{n}^{(2)^{\prime}}(\tilde{m} x)}{-\tilde{m} \varsigma_{n}^{(1)^{\prime}}(x) \varsigma_{n}^{(2)}(\tilde{m} x)+\varsigma_{n}^{(1)}(x) \varsigma_{n}^{(2)^{\prime}}(\tilde{m} x)},
\end{aligned}
$$

where $j_{n}$ is the spherical Bessel function, and $\varsigma_{n}^{(i)}$ is the Riccati-Bessel function defined in terms of the spherical Hankel function of the ith kind. The algorithms for computing the phase matrix of a particle with fixed or random orientations from $\mathbf{T}^{0}$ are the same as for the T-matrix, which can be found in references $[1,27,64]$.

In this paper, we have developed a new accurate algorithm (see Appendix) for computing the integrals involved in Eq. (8) without discretizing the integrand for numerical calculation.

\subsection{Physical-geometric Optics Approximations}

Within the framework of the PG-O approximation, the near field is computed from the geometric optics laws, and the far field is computed from the near field based on a rigorous near-to-far-field transformation. The 
aforementioned computational technique is also referred to as the physical optics approximation in some literature [e.g.,13,56]. We use the PG-O approximation throughout the paper to emphasize the role of the geometric optics laws in the near-field calculations, because, in principle, the near field can also be calculated based on the Huygens-Fresnel principle.

To compute the far field from the near field, we can use either an electric field volume integral equation [65]

$$
\vec{E}_{s c a}(\vec{r})=\frac{\exp (i k r)}{-i k r} \frac{-i k^{3}}{4 \pi} \iiint_{V}\left[\tilde{m}^{2}\left(\overrightarrow{\mathbf{r}}^{\prime}\right)-1\right]\left\{\vec{E}\left(\vec{r}^{\prime}\right)-\hat{r}\left[\hat{r} \bullet \vec{E}\left(\vec{r}^{\prime}\right)\right]\right\} \exp \left(-i k \vec{r} \bullet \vec{r}^{\prime}\right) d^{3} \vec{r}^{\prime}
$$

or the Kirchhoff surface integral equation [66],

$$
\vec{E}_{s c a}(\vec{r})=\frac{\exp (i k r)}{-i k r} \frac{k^{2}}{4 \pi} \hat{r} \times \iint_{s}\left\{\hat{n}_{s} \times \vec{E}\left(\vec{r}^{\prime}\right)-\hat{r} \times\left[\hat{n}_{s} \times \vec{H}\left(\vec{r}^{\prime}\right)\right]\right\} \exp \left(-i k \vec{r} \bullet \vec{r}^{\prime}\right) d^{2} \vec{r}^{\prime}
$$

where $\hat{r}$ is a unit vector pointing to the observation position $\vec{r}, \vec{E}$ is the electric field, $\vec{H}$ is the magnetic field, and $\hat{n}_{s}$ is a unit vector that is locally normal to the particle surface.

The amplitude scattering matrix derived from the electric field volume integral equation has been reported in previous publications [14]. Here we present equivalent but simplified formulas by using two Fresnel's transmission coefficients, instead of three transmission coefficients defined in [50], to take into account inhomogeneous waves in a large absorbing particle.

We consider a faceted particle and assume that the incoming light is incident on $N_{f}$ facets. For arbitraryshaped curved and irregular particles as found in nature, any particle geometry can be viewed as a sufficiently large number of micro facets. Therefore, the following equations are generally applicable.

Figure 1 is a schematic diagram showing the refraction of an incident wave impinging upon a local planar facet. By using the vectors in conjunction with rays defined in Fig. 1, the amplitude scattering matrix is written as 


$$
\mathbf{S}_{v o l}(\hat{r})=\sum_{j=1}^{N_{f}} D_{j} \frac{\left(\tilde{m}^{2}-1\right) \cos \theta_{t}}{N-\hat{r} \cdot \hat{e}_{t}}\left[\begin{array}{cc}
T_{\perp} \cos \theta-T_{\|} \sin \left(\theta_{i}-\tilde{\theta}_{t}\right) \hat{r} \cdot \hat{e}_{\alpha} & -T_{\|} \sin \left(\theta_{i}-\tilde{\theta}_{t}\right) \hat{r} \cdot \hat{e}_{\beta} \\
0 & T_{\perp}
\end{array}\right],
$$

where $N=N_{r}+i N_{i}$ is an effective refractive index determined by the refractive index $\tilde{m}=\tilde{m}_{r}+i \tilde{m}_{i}$ and the incident angle $\theta_{i}$, and given by [10]

$$
\begin{gathered}
N_{r}=\sqrt{\left\{\tilde{m}_{r}^{2}-\tilde{m}_{i}^{2}+\sin ^{2} \theta_{i}+\sqrt{\left.\left(\tilde{m}_{r}^{2}-\tilde{m}_{i}^{2}+\sin ^{2} \theta_{i}\right)^{2}+4 \tilde{m}_{r}^{2} \tilde{m}_{i}^{2}\right\} / 2},\right.} \\
N_{i}=\tilde{m}_{r} \tilde{m}_{i} / N_{r} .
\end{gathered}
$$

When $\tilde{m}$ is real or $\theta_{i}=0, N=\tilde{m} . \theta_{t}$ and $\tilde{\theta}_{t}$ are real and complex refractive angles, respectively, determined by Snell's law and generalized Snell’s law,

$$
\sin \theta_{i}=\tilde{m} \sin \tilde{\theta}_{t}, \quad \sin \theta_{i}=N_{r} \sin \theta_{t}
$$

In Eq. (18), $T_{\perp}$ and $T_{/ /}$are two transmission coefficients corresponding to the parallel and perpendicular polarizations given in standard texts [e.g.,66],

$$
\begin{gathered}
T_{\perp}=\frac{2 \cos \theta_{i}}{\cos \theta_{i}+\sqrt{\tilde{m}^{2}-\sin ^{2} \theta_{i}}}, \\
T_{/ /}=\frac{2 \tilde{m} \cos \theta_{i}}{\tilde{m}^{2} \cos \theta_{i}+\sqrt{\tilde{m}^{2}-\sin ^{2} \theta_{i}}},
\end{gathered}
$$

and $D_{j}$ is a shape factor that depends on the vertices $\left(\vec{r}_{q}, q=1, N_{v}\right)$ of the $\mathrm{j}$-th facet, given by [14]

$$
D_{j}=\frac{i k}{4 \pi} \sum_{q=1}^{N_{v}} \frac{\left(\vec{r}_{q+1}-\vec{r}_{q}\right) \cdot\left[\left(\hat{e}_{i}-\hat{r}\right) \times \hat{n}\right]}{\left|\hat{e}^{i}-\hat{r}\right|^{2}-\left[\left(\hat{e}_{i}-\hat{r}\right) \cdot \hat{n}\right]^{2}} \frac{\sin \left\{k\left(\hat{e}_{i}-\hat{r}\right) \cdot\left(\vec{r}_{q+1}-\vec{r}_{q}\right) / 2\right\}}{k\left(\hat{e}_{i}-\hat{r}\right) \cdot\left(\vec{r}_{q+1}-\vec{r}_{q}\right) / 2} \times \exp \left\{i k\left(\hat{e}_{i}-\hat{r}\right) \cdot\left(\vec{r}_{q+1}+\vec{r}_{q}\right) / 2\right\}
$$

As an example of application of Eq. (18) to an arbitrarily shaped particle, in section 3.1, the curved surfaces of spheres and spheroids are represented with triangular facets. Eq. (18) can be simplified at the forward and backward directions. At $\theta=0^{\circ}$, we have 


$$
\mathbf{S}_{v o l}\left(\theta=0^{\circ}\right)=\frac{k^{2} A}{2 \pi}\left[\begin{array}{ll}
1 & 0 \\
0 & 1
\end{array}\right]
$$

To derive Eq. (25) from Eq. (18), we have used the following identities:

$$
\begin{gathered}
\left(\tilde{m}^{2}-1\right) \cos \theta_{t}=\left[N-\cos \left(\theta_{t}-\theta_{i}\right)\right]\left[\cos \theta_{i}+\tilde{m} \cos \tilde{\theta}_{t}\right] \\
\tilde{m}^{2}=N^{2}-N_{i}^{2} \tan ^{2} \theta_{t}
\end{gathered}
$$

At $\left(\theta=180^{\circ}\right)$, we have

$$
\mathbf{S}_{v o l}\left(\theta=180^{\circ}\right)=\frac{k^{2}}{2 \pi} \sum_{i=1}^{N_{f}} \frac{N-\cos \left(\theta_{i}-\theta_{t}\right)}{N+\cos \left(\theta_{i}-\theta_{t}\right)}\left[\begin{array}{cc}
-1 & 0 \\
0 & 1
\end{array}\right] \cos \theta_{i} \int \exp \left(2 i \vec{e}_{i} \cdot \vec{r}\right) d^{2} \vec{r}
$$

The amplitude scattering matrix $\mathbf{S}_{\text {surf }}(\hat{r})$ derived from the Kirchhoff surface integral equation (17) is different from $\mathbf{S}_{v o l}(\hat{r})$. Specifically, $\mathbf{S}_{\text {surf }}(\hat{r})$ can be written as the sum of a diffraction amplitude matrix and a reflection amplitude matrix [50]:

$$
\begin{gathered}
\left.\mathbf{S}_{\text {surf }}(\hat{r})=\sum_{j=1}^{N_{f}} D_{j}\left\{\begin{array}{cc}
(1+\cos \theta) \cos \theta_{i}-\sin \theta_{i} \hat{r} \cdot \hat{e}_{\alpha} & \sin \theta_{i} \hat{r} \cdot \hat{e}_{\beta} \\
-\sin \theta_{i} \hat{r} \cdot \hat{e}_{\beta} & (1+\cos \theta) \cos \theta_{i}-\sin \theta_{i} \hat{r} \cdot \hat{e}_{\alpha}
\end{array}\right]+\left[\begin{array}{cc}
S_{2}^{r} & S_{3}^{r} \\
S_{4}^{r} & S_{1}^{r}
\end{array}\right]\right\}(29) \cos \theta_{i}\left(-R_{/ /} \sin ^{2} \phi_{0}+R_{\perp} \cos ^{2} \phi_{0}\right)-R_{/ /} \sin \theta \sin \theta_{i} \sin \phi_{0} \\
S_{2}^{r}=(1-\cos \theta) \cos (30) \cos \theta_{i}\left(R_{/ /}+R_{\perp}\right) \sin \phi_{0} \cos \phi_{0}+R_{/ /} \sin \theta \sin \theta_{i} \cos \phi_{0} \\
S_{3}^{r}=(1-\cos \theta \\
S_{4}^{r}=-(1-\cos \theta) \cos \theta_{i}\left(R_{/ /}+R_{\perp}\right) \sin \phi_{0} \cos \phi_{0}-R_{\perp} \sin \theta \sin \theta_{i} \cos \phi_{0} \\
S_{1}^{r}=(1-\cos \theta) \cos \theta_{i}\left(R_{/ /} \cos ^{2} \phi_{0}-R_{\perp} \sin ^{2} \phi_{0}\right)-R_{\perp} \sin \theta \sin \theta_{i} \sin \phi_{0}
\end{gathered}
$$

where $R_{/ /}$and $R_{\perp}$ are the reflection coefficients, given by [66]

$$
R_{/ /}=\frac{\tilde{m} \cos \theta_{i}-\cos \tilde{\theta}_{t}}{\tilde{m} \cos \theta_{i}+\cos \tilde{\theta}_{t}}
$$




$$
R_{\perp}=\frac{\cos \theta_{i}-\tilde{m} \cos \tilde{\theta}_{t}}{\cos \theta_{i}+\tilde{m} \cos \tilde{\theta}_{t}}
$$

and $\phi_{0}$ is determined through

$$
\phi_{0}=\operatorname{atan} 2\left(\hat{r} \cdot \hat{e}_{\alpha} / \hat{r} \cdot \hat{e}_{\beta}\right)
$$

In Eq. (36), atan 2 is similar to the atan function, except that $\phi_{0}$ is specified with its correct quadrant.

Diffraction by a disk or plate is a classical subject in the history of electromagnetic wave theory. The simplest case of the diffraction and reflection calculation is the normal incidence on a disk. In this case, there is only one facet that intercepts the incident wave. The PG-O approximations do not depend on the disk thickness and are solely related to the shape and size of the facet. The amplitude scattering matrices $\mathbf{S}_{\text {vol }}(\hat{r})$ and $\mathbf{S}_{\text {surf }}(\hat{r})$ are dramatically simplified, written as

$$
\begin{gathered}
\mathbf{S}_{v o l}(\hat{r})=\frac{2(\tilde{m}-1)}{\tilde{m}-\cos \theta}\left[\begin{array}{cc}
\cos \theta & 0 \\
0 & 1
\end{array}\right] D, \\
\mathbf{S}_{\text {surf }}(\hat{r})=\frac{2}{\tilde{m}+1}\left[\begin{array}{cc}
1+\tilde{m} \cos \theta & 0 \\
0 & \tilde{m}+\cos \theta
\end{array}\right] D .
\end{gathered}
$$

The succinctness of the above two equations enables an intuitive grasp of the characteristics of the two different physical-optics formalisms. For example, we have the following three scenarios:

(1) When $\tilde{m}=1$, there is no scattering. In this case, Eq. (37) is zero as expected; however, Eq. (38) is nonzero. The reason is that the Kirchhoff equation is now applied to a non-exiting facet (actually, the Kirchhoff equation has to be applied to a closed surface instead of a facet to give zero scattering). As it turns out, the Kirchhoff equation is cumbersome for calculating diffraction and reflection as the refractive index approaches unity.

(2) When $\tilde{m} \neq 1$, Eqs. (37) and (38) are identical at the exact forward and backward directions, but are different at all other angles. For example, at $\theta=90^{\circ}$, we have

$$
\mathbf{S}_{v o l}(\hat{r})=\frac{2(\tilde{m}-1)}{\tilde{m}}\left[\begin{array}{ll}
0 & 0 \\
0 & 1
\end{array}\right] D,
$$




$$
\mathbf{S}_{\text {surf }}(\hat{r})=\frac{2}{\tilde{m}+1}\left[\begin{array}{cc}
1 & 0 \\
0 & \tilde{m}
\end{array}\right] D
$$

(3) When $\mathrm{m}>>1, \mathbf{S}_{\text {vol }}(\hat{r})$ and $\mathbf{S}_{\text {surf }}(\hat{r})$ asymptotically approach

$$
\mathbf{S}_{v o l}(\hat{r})=\mathbf{S}_{\text {surf }}(\hat{r})=2\left[\begin{array}{cc}
\cos \theta & 0 \\
0 & 1
\end{array}\right] D
$$

Therefore, $\mathbf{S}_{\text {vol }}(\hat{r})$ and $\mathbf{S}_{\text {surf }}(\hat{r})$ yield closer results for all scattering angles as the modulus of the refractive index becomes larger. It is evident that the asymptotic phase function is symmetric with respect to $\theta=90^{\circ}$.

\section{Numerical Results and Discussion}

\subsection{Spheres, Spheroids and Disks}

As the first numerical experiment, we compute the diffraction and reflection pattern associated with a sphere, an oblate spheroid and a prolate spheroid. For spheroids, we further assume that the incident direction is aligned with the symmetry axis and the projected area is the same as that of a sphere. According to the Babinet principle or the Kirchhoff surface integral equation, the diffraction patterns for the three geometries are identical. In the PG-O approximation, the surfaces of the three particles are faceted with a large number of triangles so that the formulas outlined in Section 2 are readily applicable.

Figure 2 shows the comparison of phase functions computed from the Debye series and the PG-O approximations based on the Kirchhoff surface integral equation and the electric field volume integral equation. The two PG-O approximations yield similar features of the phase function, but fail to predict the oscillation amplitude and the locations of peaks and minima. Although the three geometries have the same boundary that separates the illuminated and shadowed sides, the diffraction patterns from the Debye series are different. This feature can be explained by different edge-effect impacts on the diffraction pattern and associated interference effect between diffraction and reflection. The edge-effect efficiencies for three different cases are also given in Fig. 2. The edge-effect efficiency is the largest for prolate spheroids and the smallest for oblate spheroids. It is interesting to note that the first four minima of the PG-O phase function for oblate spheroids are closer to the rigorous results than those for spheres and prolate spheroids. It seems that 
the larger the edge-effect efficiency, the more significant the deviation of peak locations from the Fraunhofer diffraction pattern. If we concentrate on the forward scattering direction, the phase function of the PG-O approximations is less than that of the Debye series. This phenomenon is also due to the missing edge effect in the PG-O approximations. At scattering angles between 40 and 100 degrees, the agreement degrades because the contributions from both tunneling and the Fraunhofer diffraction are small so that the phase change of the total field deviates significantly from the counterpart associated with the diffraction component. However, the reflection patterns for all the cases obtained from the PG-O approximations are quite close to the Debye solutions. From this comparison, we learn that the edge-effect impact on the phase function is mainly observed at scattering angles smaller than 90 degrees. From Fig. 2, it is difficult to determine the best PG-O approach among all the approximations, because the phase function at a specific scattering angle has contributions from many facets. The superposition process probably suppresses the differences between the two PG-O approximations.

The second numerical experiment is the disk scattering problem. If the light impinges the basal face of a disk, the PG-O approximations are remarkably simplified so differences between the two PG-O approximations can be well explained. For a triangular prism, in the Debye series calculation, the height of the prism is equal to $k a$. Fig. 3 compares the phase functions computed from the two PG-O approximations. The phase functions are averaged with respect to the azimuthal scattering angle. As can be clearly seen, the two approximations are exactly the same in the forward and backward scattering direction, but differ in side scattering directions, in particular for smaller refractive indices. The PG-O approximation based on the electric field volume integration is better than the PG-O based on the Kirchhoff surface integration, in comparison with their rigorous counterparts. We note that, unlike the other regular polygons, the oscillations of the scattering pattern for a regular triangle are significantly suppressed so that we can see more clearly the difference trend of the phase function with respect to the scattering angle (results for the other regular polygons are not shown). Another virtue of the second numerical experiment is that the reflection in a geometric-optics sense is in the backward direction. In this case, the forward scattering pattern can be viewed as "solely" from diffraction. Similar to findings obtained in Fig. 2, we observe that the backscattering pattern from reflection is more accurate than the forward scattering pattern. We note that tunneling efficiencies for disks with normal incidence are much larger than those for spheres and spheroids in Fig. 2. We also observe that the forward scattering differences are substantial in all the cases. Furthermore, an ratio of the forward scattering cross sections by the two methods, i.e., $f(0)$ (Debye/PG-O), is shown in each panel of Fig. 3. 
As predicted from the PG-O approximations, the diffraction pattern depends only on the shape and size of the geometric cross section. Based on the Debye series, we are able to investigate the dependence of diffraction on refractive indices and thereby quantify the PG-O accuracy. Figure 4 compares the diffraction-plus-reflection phase functions computed from the zeroth-order Debye series for particles with different degrees of absorption. We assume the real part of the refractive index to be constant with the imaginary part increasing from 0 to 0.5 . As demonstrated from the comparison, the oscillation pattern is similar for all the refractive indices with different imaginary parts. A large imaginary part leads to higher backscattering. For forward scattering, the amplitude of oscillation is smaller when the particle is more absorptive. We notice that the locations of peaks and minima remain the same.

Next, we examine how the edge effect influences the diffraction pattern for particles with different aspect ratios. We focus on strongly absorptive particles, because absorption does not change the locations of peaks and minima. In this case, we can apply the DDA method [22] to compute the optical properties of particles with extreme aspect ratios (Fig. 5). We consider a cube and a plate, and assume again that light is incident normally to the basal facet. Four size parameters $(40,80,120$, and 160$)$, defined in terms of the width of the particle, are selected. The aspect ratios of the plate (height/width) corresponding to the aforementioned size parameters are $0.2,0.1,0.1$ and 0.05 . As expected, the cube and the plate have the same results from the PG$\mathrm{O}$ approximation because the shadow is the same (the PG-O volume curve corresponds to both cube and plate). Also, the reflection pattern is insensitive to the particle thickness, and the peaks and minima from the PG-O approximation match the DDA simulations very well. However, the oscillation amplitude and the locations of peaks and minima for forward scattering (scattering angles less than 90 degrees) are sensitive to the particle thickness. For a plate, diffraction patterns with forward scattering match very well with those from the DDA results. From this comparison, we postulate that as the thickness approaches zero, the PG-O approximate results will be close to their rigorous counterparts at all scattering angles, because the diffraction of light by a disk of zero thickness is equivalent to a circular aperture of the same radius in an infinite plane.

From the numerical experiments presented in this section, we find that the edge effect has more influence on the forward scattering phase function than on the backscattering phase function. The edge effect contribution can be dominant at the near-forward scattering direction. It is important to point out that the phase functions 
presented in Fig. 2 - Fig.5 have not been normalized. If normalized phase functions are used for comparison, the reflection patterns will be shifted upward in comparison with Debye's reflections. Bearing in mind these findings, the next section studies the optical properties of faceted particles with fixed or random orientations.

\subsection{Faceted particles with fixed and random orientations}

In this section, we compute the phase matrix for faceted particles. For particles with fixed orientations, the phase matrix is averaged with respect to the azimuthal angle of scattering planes.

Figure 6 shows the diffraction plus reflection pattern of hexagonal ice crystals with fixed orientations. The refractive index is 1.3. Four size parameters $(20,30,40$, and 50) are selected for comparison purposes. For each size parameter, the results for three orientations are presented. We again observe that the PG-O approximation has better accuracy in computing the reflection part of the phase function. We can clearly see the peaks from the specular reflection. When the size parameter increases, the peaks become narrower because there is less spreading of reflection energy to the near by scattering angles. The PG-O approximations fail to reproduce the oscillations in the diffraction pattern and result in smaller forward scattering cross section due to the missing edge effect.

Figure 7 shows the phase matrix for randomly oriented hexagonal ice crystals. The particle is non-absorptive and has a size parameter of 50 . The tunneling efficiency is 0.0677 , a relatively small value. We can see that the diffraction pattern looks similar if the condition of random orientation is considered. The phase functions computed from the Debye and PG-O volume methods resemble each other better than the case of fixed orientations. The $\mathrm{P}_{22} / \mathrm{P}_{11}$ ratio from the Debye method is found to be close to unity, but the PG-O volume result are even closer to unity. The differences of $-\mathrm{P}_{12} / \mathrm{P}_{11}$ computed from the two methods are relatively larger when the scattering angle is less than 90 degrees. The comparisons of the other elements $\left(P_{33} / P_{11}, P_{44} / P_{11}\right.$, and $\mathrm{P}_{34} / \mathrm{P}_{11}$ ) show satisfactory agreement. We included the PG-O surface results for comparison and found that the PG-O volume method is much better than the PG-O surface method. 
Figure 8 is similar to Fig. 7 except that the refractive index is $1.3+i 0.5$. Higher absorption leads to smaller tunneling efficiency. We observe that the $-P_{12} / P_{11}$ comparison is much better and $P_{34} / P_{11}$ now has large values in comparison with the case shown in Fig. 7. The $P_{22} / P_{11}$ comparison does not show improvement, probably because the values are close to unity.

To understand the shape dependence of the tunneling effect on the optical properties, we compute the diffraction plus reflection phase function as well as tunneling efficiencies for a few representative faceted particles. Figure 9 shows the comparison of the phase functions computed from the Debye series and the PG-O approximations for a cube, an octahedron and a dodecahedron. The particles are assumed to be randomly oriented. The refractive index is 1.3. As the size parameter increases, we observe better agreement with respect to the near forward scattering including the first diffraction peak. The tunneling efficiency is a good indicator of the accuracy of the PG-O approximation in computing the diffraction pattern. For example, for a cube, at size parameter $k R=30$, the tunneling efficiency is 0.0366 . In this case, the forward scattering phase functions computed from the Debye series and the PG-O approximation closely resemble each other. Therefore, the dependence of the tunneling efficiency on the size parameter can be employed to understand the transition from the full electromagnetic wave theory to the PG-O approximations.

Table 2 tabulates the tunneling efficiencies for a sphere and randomly oriented nonspherical particles. In general, the tunneling efficiencies decrease as the size parameter increases, except for fairly small size parameters for which the ray concept fails and the tunneling concept is vague. It seems to be "reliable" to conclude that "the larger the size parameter, the better the PG-O accuracy" for randomly oriented nonspherical particles. We note that for a cube, the tunneling efficiencies are much smaller than for the other particle shapes. For particles with fixed orientation, the dependence of the tunneling efficiency on the particle shape and orientation may have different characteristics. For example, in the case of cylinders with quasinormal incidence to the basal face, the tunneling efficiency is sensitive to the particle aspect ratio. If the particle size increases along the dimension in the incident direction, the PG-O accuracy deteriorates in computing the diffraction.

\section{Summary and Conclusions}


In summary, we have separated diffraction and reflection from scattering by using a rigorous approach based on the Debye series. The separation enables us to have a better understanding of the edge/tunneling effect on the extinction efficiency and the phase matrix. Furthermore, we have examined the accuracy of the PG-O approximations in computing the diffraction plus reflection by nonspherical particles through comparing the PG-O results with their rigorous counterparts provided by the Debye series. The major findings of this study are summarized as follows:

(1) Although a rigorous definition of diffraction for a three-dimensional particle is nonexistent, diffraction together with reflection can be defined without ambiguity. The zeroth-order Debye series provides a "tractable" definition of diffraction by a disk of finite thickness by assuming reflection is in backscattering directions. The Fraunhofer diffraction, reflection and tunneling are underlying physical mechanisms to understand diffraction plus reflection determined from the zeroth-order Debye series.

(2) The statement "the larger the size parameter, the better the PG-O accuracy" is inaccurate. The particle geometry (e.g., the aspect ratio) and the direction of incident light are also critical factors that affect the accuracy of the PG-O approximation. If the particle size increases only in the direction of the incident light, the PG-O accuracy decreases. For particular cases associated with oriented particles, PGO fails to reproduce the oscillation pattern in the polarization-related phase matrix elements regardless of the size parameter.

(3) For randomly oriented nonspherical particles, the tunneling effect may have little effect on the diffraction and reflection pattern particularly when the size parameter is large. The tunneling may affect high-order scattering associated with the higher-order terms in the Debye series, which have not yet been systematically investigated for nonspherical particles.

(4) We demonstrate that the electric field volume-integral equation is usually much more accurate than the surface integral equation, in particular, at side scattering angles when the refractive index is moderate and small. This conclusion does not mean that it is impossible to find cases for which the results from the surface-integral equation are closer to rigorous counterparts than the results from the electric field volume-integral equation.

(5) The tunneling efficiencies are tabulated for several canonical nonspherical particles. For randomly oriented particles, the tunneling efficiency decreases with respect to the size parameter. In addition, as the imaginary part of the refractive indices increases, the tunneling efficiency decreases. 
Based on the aforesaid findings, the PG-O approximations can provide the phase matrix of randomly oriented particles with reasonable accuracy. For a particle with a fixed orientation, the PG-O approximation is applicable to the computation of the phase function when the tunneling efficiency is small. Furthermore, it is necessary to better quantify the uncertainty associated with the application of the PG-O approximation to the computation of the polarization-related phase matrix elements (e.g., the depolarization ratio). In particular, further efforts are required to obtain the higher-order Debye series in order to understand the PG-O accuracy in computing the contribution to the phase matrix from higher-order transmitted light.

\section{Acknowledgement}

This study was supported by an NSF grant (AGS-1338440). The authors thank M. A. Yurkin and A. G. Hoestra for use of their ADDA code. A major portion of the simulations was carried out at the Texas A\&M University Supercomputing Facilities. 


\section{Appendix: An analytical algorithm to solve U-matrix in Eq. (8)}

In the cases of axially symmetric particles, the refractive index is non-unity in piecewise intervals. Assume the refractive index is non-unity in a range $\left[\theta_{1}, \theta_{2}\right]$, the numerical computations in Eq. (8) can be simplified by using the following identities,

$$
\begin{gathered}
\int_{\theta_{1}}^{\theta_{2}} U^{2} \sin \theta d \theta=i m \delta_{m m^{\prime}}\left[d_{0 m}^{n}\left(\theta_{2}\right) d_{0 m}^{n^{\prime}}\left(\theta_{2}\right)-d_{0 m}^{n}\left(\theta_{1}\right) d_{0 m}^{n^{\prime}}\left(\theta_{1}\right)\right], \\
\int_{\theta_{1}}^{\theta_{2}} U^{1} \sin \theta d \theta=\delta_{m m^{\prime}}\left[\tau_{m^{\prime} n^{\prime}}(\theta) d_{0 m}^{n}(\theta) \sin \theta\right] \mid \theta_{\theta_{1}}+\sqrt{\frac{n(n+1)}{n^{\prime}\left(n^{\prime}+1\right)}} \int_{\theta_{1}}^{\theta_{2}} U^{3} \sin \theta d \theta .
\end{gathered}
$$

Eq. (42) is trivial and Eq. (43) is obtained by employing Eq. B(25) in [67]. With Eqs. (42) and (43), only the following integration in Eq. (8) needs special treatment:

$$
\int_{\theta_{1}}^{\theta_{2}} U^{3} \sin \theta d \theta
$$

To solve Eq. (44), we have to compute the following integral,

$$
I_{m}^{n n^{\prime}}=\int_{\theta_{1}}^{\theta_{2}} d_{m 0}^{n}(\theta) d_{m 0}^{n^{\prime}}(\theta) \sin \theta d \theta
$$

When $n=n^{\prime}$, let $I_{m}^{n}=I_{m}^{n n}$ and it can be proved that

$$
I_{0}^{n}=\left.\sum_{k=0}^{2 n}\left(\begin{array}{ccc}
n & n & 2 k \\
0 & 0 & 0
\end{array}\right)^{2}\left(d_{2 k+1,0}^{n}-d_{2 k-1,0}^{n}\right)\right|_{\theta_{1}} ^{\theta_{2}}
$$

where $\left(\begin{array}{ccc}n & n & 2 k \\ 0 & 0 & 0\end{array}\right)$ is Wigner 3j-symbol. In deriving Eq. (46), we have used the expansion of the product of two Legendre's polynomials in terms of Legendre's polynomials presented in Adams [68]. $I_{m}^{n}(m>0)$ can be computed from the following recurrence relationships 


$$
I_{m}^{n}=\frac{1}{n+m}\left[-(n-m+1) I_{m-1}^{n}+(n-m) I_{m}^{n-1}+(n+m-1) I_{m-1}^{n-1}\right],
$$

which is obtained from the following recurrence relation of associated Legendre's polynomials:

$$
\left(P_{l}^{m+1}\right)^{2}+(l-m)^{2}\left(P_{l}^{m}\right)^{2}=\left(P_{l-1}^{m+1}\right)^{2}+(l+m)^{2}\left(P_{l-1}^{m}\right)^{2} .
$$

$I_{m}^{n n^{\prime}}\left(n \neq n^{\prime}\right)$ can be analytically given as

$$
I_{m}^{n n^{\prime}}=\frac{\left[\sin ^{2} \theta\left(\pi_{m n}(\theta) \tau_{m n^{\prime}}(\theta)-\pi_{m n^{\prime}}(\theta) \tau_{m n}(\theta)\right)\right]_{\theta_{1}}^{\theta_{2}}}{m\left[n(n+1)-n^{\prime}\left(n^{\prime}+1\right)\right]},
$$

which is obtained from associated Legendre differential equation.

Based on Eqs.(42)-(49), Eq. (8) can be computed more efficiently and accurately than the use of numerical integration technique, such as Gaussian quadrature. 


\section{References}

1. Mishchenko MI, Hovenier JW, Travis LD, editors, Light scattering by nonspherical particles: theory, measurements, and applications. Academic; 2000.

2. Kokhanovsky A, editor. Light scattering reviews: single and multiple light scattering. Springer; 2006.

3. Yang P, Liou KN, Bi L, Liu C, Yi B, Baum BA. On the radiative properties of ice clouds: light scattering, remote sensing, and radiation parameterization. Adv Atmos Sci 2015; 32(1): 32-63.

4. $\quad$ van de Hulst HC. Light scattering by small particles. New York: Dover; 1981.

5. Nussenzveig HM. Diffraction effects in semiclassical scattering. Cambridge University; 1992.

6. Mishchenko MI, Tishkovets VP, Travis LD, et al. Electromagnetic scattering by a morphologically complex object: fundamental concepts and common misconceptions. J Quant Spectrosc Radiat Trans 2011; 112:671-92.

7. Takano Y, Liou KN. Solar radiative transfer in cirrus clouds. Part I: single-scattering and optical properties of hexagonal ice crystals. J Atmos Sci 1989;46:3-19.

8. Macke A. Scattering of light by polyhedral ice crystals. Appl Opt 1993; 32: 2780-88.

9. Muinonen K. Scattering of light by crystals: a modified Kirchhoff approximation. Appl Opt 1989; 28: 3044-50.

10. Yang P, Liou KN. Geometric-optics-integral-equation method for light scattering by nonspherical ice crystals. Appl Opt 1996; 35: 6568-84.

11. Yang P, Liou KN. Light scattering by hexagonal ice crystals: solutions by a ray-by-ray integration algorithm. J Opt Soc Am A 1997; 14: 2278-89.

12. Borovoi AG, Grishin IA. Scattering matrices for large ice crystal particles. J Opt Soc Am A 2003;20:2071-80.

13. Borovoi AG, Konoshonkin A, Kustova N. The physical-optics approximation and its application to light backscattering by hexagonal ice crystals. J Quant Spectrosc Radiat Transf 2014; 146, 181-89.

14. Bi L, Yang P, Kattawar GW, Hu Y, Baum BA. Scattering and absorption of light by ice particles: solution by a new physical-geometric optics hybrid method. J Quant Spectrosc Radiat Transf 2011; 112:1492-508.

15. Ren KF, Rozé C, Girasole T. Scattering and transversal divergence of an ellipsoidal particle by using Vectorial Complex Ray Model. J Quant Spectrosc Radiat Transfer 2012; 113:2419-2423. 
16. Yee SK. Numerical solution of initial boundary value problems involving Maxwell's equations in isotropic media. IEEE Trans Antennas Propag 1966; 14: 302-7.

17. Yang P, Liou KN. Finite-difference time domain method for light scattering by small ice crystals in three-dimensional space. J Opt Soc Amer 1996; 13(10): 2072-85.

18. Sun W, Fu Q, Chen Z. Finite-difference time-domain solution of light scattering by dielectric particles with perfectly matched layer absorbing boundary conditions. Appl Opt 1999; 38: 314151.

19. Liu C, Panetta RL, Yang P. Application of the pseudo-spectral time domain method to compute particle single-scattering properties for size parameters up to 200. J Quant Spectrosc Radiat Transf 2012; 113: 1728-40.

20. Purcell EM, Pennypacker CR. Scattering and absorption of light by nonspherical dielectric grains. Astrophys J 1973;186: 705-14.

21. Draine BT. The discrete-dipole approximation and its application to interstellar graphite grains. Astrophys J 1988; 333: 848-72.

22. Yurkin MA, Maltsev VP, Hoekstra AG. The discrete dipole approximation for simulation of light scattering by particles much larger than the wavelength. J Quant Spectrosc Radiat Transf 2007; 106: 546-57.

23. Waterman PC. Matrix formulation of electromagnetic scattering. Proc IEEE 1965; 53: 805-12.

24. Mishchenko MI, Travis LD, Mackowski DW, T-matrix computations of light scattering by nonspherical particles: a review. J Quant Spectrosc Radiat Transf 1996; 55: 535-75.

25. Mackowski DW, Mishchenko MI. Calculation of the T matrix and the scattering matrix for ensembles of spheres. J Opt Soc Am A 1996;13: 2266-78.

26. Havemann S, Baran AJ. Extension of T-matrix to scattering of electromagnetic plane waves by non-axisymmetric dielectric particles: application to hexagonal ice cylinders. J Quant Spectrosc Radiat Transf 2001; 70: 139-58.

27. Bi L, Yang P, Accurate simulation of the optical properties of atmospheric ice crystals with invariant imbedding T-matrix method. J Quant Spectrosc Radiat Transf 2014; 138:17-35.

28. Sun W, Lin B, Hu Y, Wang Z, Fu Y, Feng Q, Yang P. Side-face effect of a dielectric strip on its optical properties. IEEE Trans Geosci Remote Sens. 2008; 46:2337-44.

29. Yang M, Wu Y, Sheng X, Ren KF. Comparison of scattering diagrams of large nonspherical particles calculated by VCRM and MLFMA. J Quant Spectrosc Radiat Transf 2015; 162:143-53. 
30. Um J, McFarquhar GM. Formation of atmospheric halos and applicability of geometric optics for calculating single-scattering properties of hexagonal ice crystals: Impacts of aspect ratio and ice crystal size. J Quant Spectrosc Radiat Transf 2015;165:134-52.

31. Bi L, Yang P. Physical-geometric optics hybrid methods for computing the scattering and absorption properties of ice crystals and dust aerosols. In: Kokhanovsky A, editor. Light Scattering Reviews, vol. 8. Chichester, UK: Springer-Praxis Publishing; 2013 (69-114).

32. Bi L, Yang P, Liu C, Yi B, Baum BA, van Diedenhoven B, Iwabuchi H. Assessment of the accuracy of the conventional ray-tracing technique: Implications in remote sensing and radiative transfer involving ice clouds. J Quant Spectrosc Radiat Transf 2014, 146: 158-74.

33. Zhou C, Yang P. Backscattering peak of ice cloud particles. Opt Express 2015; 23: 11995-2003.

34. Debye P. Das elektromagnetische Feld um einen Zylinder und die Theorie des Regenbogens. Phys Zeit 1908; 9: 775-8.

35. van de Pol B, Bremmer H. Diffraction of electromagnetic waves from an electric point source round a finiely conducting sphere, with application to radiotelegraphy and the theory of the rainbow. Phil. Mag. 1937; 24: 141-176.

36. Hovenac EA, Lock JA. Assessing the contributions of surface waves and complex rays to far-field Mie scattering by use of the Debye series. J Opt Soc Am A 1992; 9:781-95.

37. Nussenzveig HM. Complex angular momentum theory of the rainbow and the glory. J Opt Soc Am A 1979; 69(8):1068-79.

38. Lock JA, Laven P. Mie scattering in the time domain. Part I. The role of surface waves. J Opt Soc Am A 2011; 28(6): 1086-95.

39. Lock JA, Laven P. Mie scattering in the time domain. Part II. The role of diffraction. J Opt Soc Am A 2011; 28(6):1096-106.

40. Lock JA, Jamison JM, Lin CY. Rainbow scattering by a coated sphere. Appl Opt 1994; 33: 467790.

41. Lock JA, Debye series analysis of scattering of a plane wave by a spherical Bragg grating. Appl Opt 2005; 44: 5594-603.

42. Xu F, Lock JA, Tropea C. Debye series for light scattering by a spheroid. J Opt Soc Am A 2010; 27: 671-86.

43. Xu F, Lock JA, Gouesbet G. Debye series for light scattering by a nonspherical particle. Phys Rev A 2010; 81:043824. 
44. Bi L, Yang P, Kattawar GW, Mishchenko MI. Optical tunneling by arbitrary macroscopic threedimensional objects. Phys Rev A, 2015; 92: 013814.

45. Johnson BR. Invariant imbedding T-matrix approach to electromagnetic scattering. Appl Opt 1988;27:4861-73.

46. Bi L, Yang P, Kattawar GW, Mishchenko MI. Efficient implementation of the invariant imbedding T-matrix method and the separation of variables method applied to large nonspherical inhomogeneous particles. J Quant Spectrosc Radiat Transf 2013; 116: 169-83.

47. Bi L, Yang P, Kattawar GW, Mishchenko MI. A numerical combination of extended boundary condition and invariant imbedding method applied to light scattering by large spheroids and cylinders. J Quant Spectrosc Radiat Transf 2013; 123:17-22.

48. Bi L, Yang P. Modeling of light scattering by biconcave and deformed red blood cells with the invariant imbedding T-matrix method. J Biomedical Optics, 2013; 18(5): 055001.

49. Bi L, Yang P. Impact of calcification state on the inherent optical properties of Emiliania huxleyi coccoliths and coccolithophores. J Quant Spectrosc Radiat Transf 2015; 155:10-21.

50. Yang P, Gao BC, Baum BA, Wiscombe WJ, Mischenko MI, Winker DM, Nasiri SL. Asymptotic solutions of optical properties of large particles with strong absorption. Appl Opt 2001; 40: 153247.

51. Bi L, Yang P, Kattawar GW, Hu Y, Baum BA. Diffraction and external reflection by dielectric faceted particles. J Quant Spectrosc Radiat Transf 2011; 112: 163-73.

52. Hesse E, Collier CT, Penttilä A, Nousiainen T, Ulanowski Z, Kaye PH. Modelling light scattering by absorbing smooth and slightly rough facetted particles. J Quant Spectrosc Radiat Transf 2015; 157:71-80.

53. Born M, Wolf E. Principles of Optics. Oxford: Pergamon Press; 1957.

54. Laven P. Separating diffraction from scattering: the million-dollar challenge. J Nanophotonics 2010; 4:041593.

55. Keller JB. Geometric theory of diffraction. J Opt Soc Amer 1962; 52(2):116-30.

56. Ufimtsev PY. Fundamentals of the physical theory of diffraction. New Jersey: Wiley; 2007.

57. Groth SP, Hewett DP, Langdon S. Hybrid numerical-asymptotic approximation for high-frequency scattering by penetrable convex polygons. IMA J Appl Math 2015; 80(2):324-353 
58. Yang P, Feng Q, Hong G, Kattawar GW, Wiscombe WJ, Mishchenko MI, Dubovik O, Laszlo I, Sokolik IN. Modeling of the scattering and radiative properties of nonspherical dust particles. J Aerosol Sci, 2007; 38, 995-1014.

59. Liou KN, Takano Y,Yang P. On geometric optics and surface waves for light scattering by spheres. J Quant Spectrosc Radiat Transf, 2010; 111, 1980-89.

60. Liou KN, Takano Y, Yang P. Light absorption and scattering by aggregates: Application to black carbon and snow grain. J. Quant. Spectrosc. Radiat. Transf, 2011; 112:1581-94.

61. Bi L, Yang P. High-frequency extinction efficiencies of spheroids: rigorous T-matrix solutions and semi-empirical approximations. Opt Express 2014; 22: 10270-93.

62. Fock VA. Electromagnetic diffraction and propagation problems. Pergamon: Oxford;1965.

63. Yi JM, Cuche A, de León Pérez, Degiron A, Laux E, Devaux E, Genet C, Alegret J, Moreno LM, Ebbesen TW. Diffraction regimes of single holes. Phys Rev Lett 2012; 109: 023901.

64. Mishchenko MI. Calculation of the amplitude matrix for a nonspherical particle in a fixed orientation. Appl Opt, 2000; 39:1026-31.

65. Saxon DS. Lectures on the scattering of light. Los Angeles: University of California; 1955.

66. Jackson JD. Classical Electrodynamics. $3^{\text {rd }}$ ed. New York: Academic Press; 1998.

67. Mishchenko MI, Travis LD, Lacis AA. Scattering, absorption and emission of light by small particles. Cambridge: Cambridge University Press; 2002.

68. Adams JC. On the expression of the product of any two Legendre's coefficients by means of a series of Legendre’s coefficients. Proc R Soc Lond, 1878; 27:63-71. 
Table 1

Extinction mechanisms considered by different methods to compute the extinction efficiency.

\begin{tabular}{lccc}
\hline Extinction Mechanisms & T-matrix & Oth-order Debye & PG-O \\
\hline The Fraunhofer diffraction & Yes & Yes & Yes \\
Blocking & Yes & Yes & Yes \\
Interference with transmission & Yes & No & Yes \\
Edge effect & Yes & Yes & No \\
Surface waves & Yes & No & No \\
\hline
\end{tabular}




\section{Table 2}

Tunneling efficiencies of a few representative faceted particles that are assumed to be randomly oriented. The results for a sphere are included for comparison.

\begin{tabular}{|c|c|c|c|c|c|c|}
\hline \multirow{3}{*}{ Shape } & \multirow{3}{*}{$\tilde{m}$} & \multicolumn{5}{|c|}{ Size Parameter } \\
\hline & & 10 & 15 & 20 & 25 & 30 \\
\hline & & \multicolumn{5}{|c|}{ Tunneling Efficiency } \\
\hline \multirow{2}{*}{ Octahedron } & 1.3 & 0.6011 & 0.4247 & 0.3520 & 0.3004 & 0.2682 \\
\hline & $1.3+i 0.5$ & 0.3039 & 0.2704 & 0.2512 & 0.2305 & 0.2206 \\
\hline \multirow{2}{*}{ Cube } & 1.3 & 0.2775 & 0.1380 & 0.0918 & 0.0469 & 0.0366 \\
\hline & $1.3+i 0.5$ & 0.0172 & 0.0219 & 0.0209 & 0.0197 & 0.0174 \\
\hline \multirow{2}{*}{ Dodecahedron } & 1.3 & 0.3850 & 0.2448 & 0.1874 & 0.1450 & 0.1170 \\
\hline & $1.3+i 0.5$ & 0.1542 & 0.1231 & 0.0957 & 0.0799 & 0.0693 \\
\hline \multirow{2}{*}{ Hexagon } & 1.3 & 0.3225 & 0.1826 & 0.1196 & 0.0880 & 0.0731 \\
\hline & $1.3+i 0.5$ & 0.0833 & 0.0716 & 0.0616 & 0.0513 & 0.0463 \\
\hline \multirow{2}{*}{ Droxtal } & 1.3 & 0.4238 & 0.2830 & 0.2155 & 0.1755 & 0.1422 \\
\hline & $1.3+i 0.5$ & 0.2116 & 0.1668 & 0.1346 & 0.1147 & 0.1006 \\
\hline
\end{tabular}




\begin{tabular}{|l|l|l|l|l|l|l|}
\hline \multirow{3}{*}{ Sphere } & 1.3 & 0.4991 & 0.3604 & 0.2892 & 0.2450 & 0.2146 \\
\cline { 2 - 7 } & $1.3+\mathrm{i} 0.5$ & 0.3096 & 0.2537 & 0.2176 & 0.1923 & 0.1734 \\
\hline
\end{tabular}

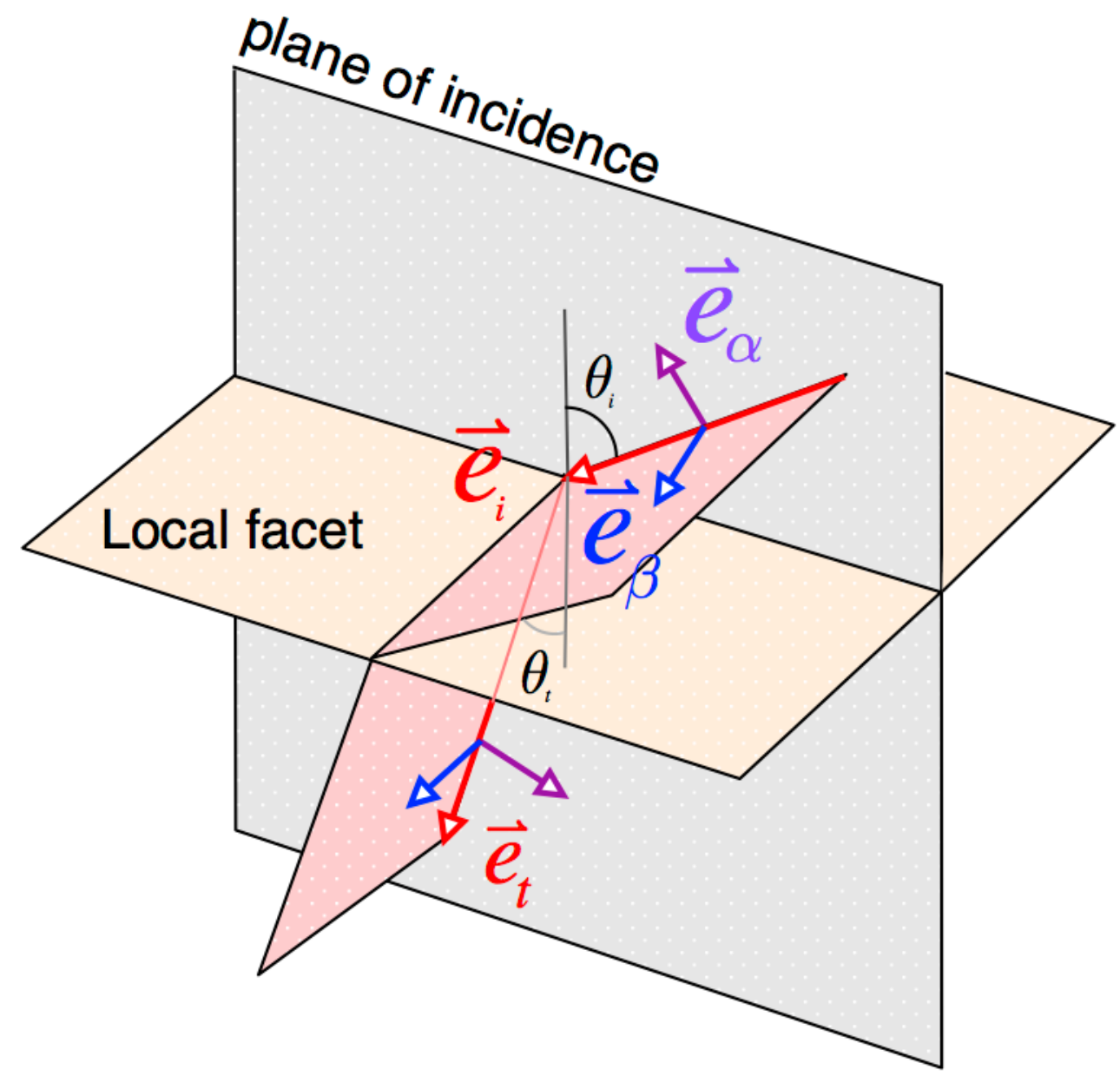

Fig. 1. Conceptual figure to illustrate the refraction of an incident wave upon a local facet. Note that $\vec{e}_{\beta} \times \vec{e}_{\alpha}=\vec{e}_{i}$. 


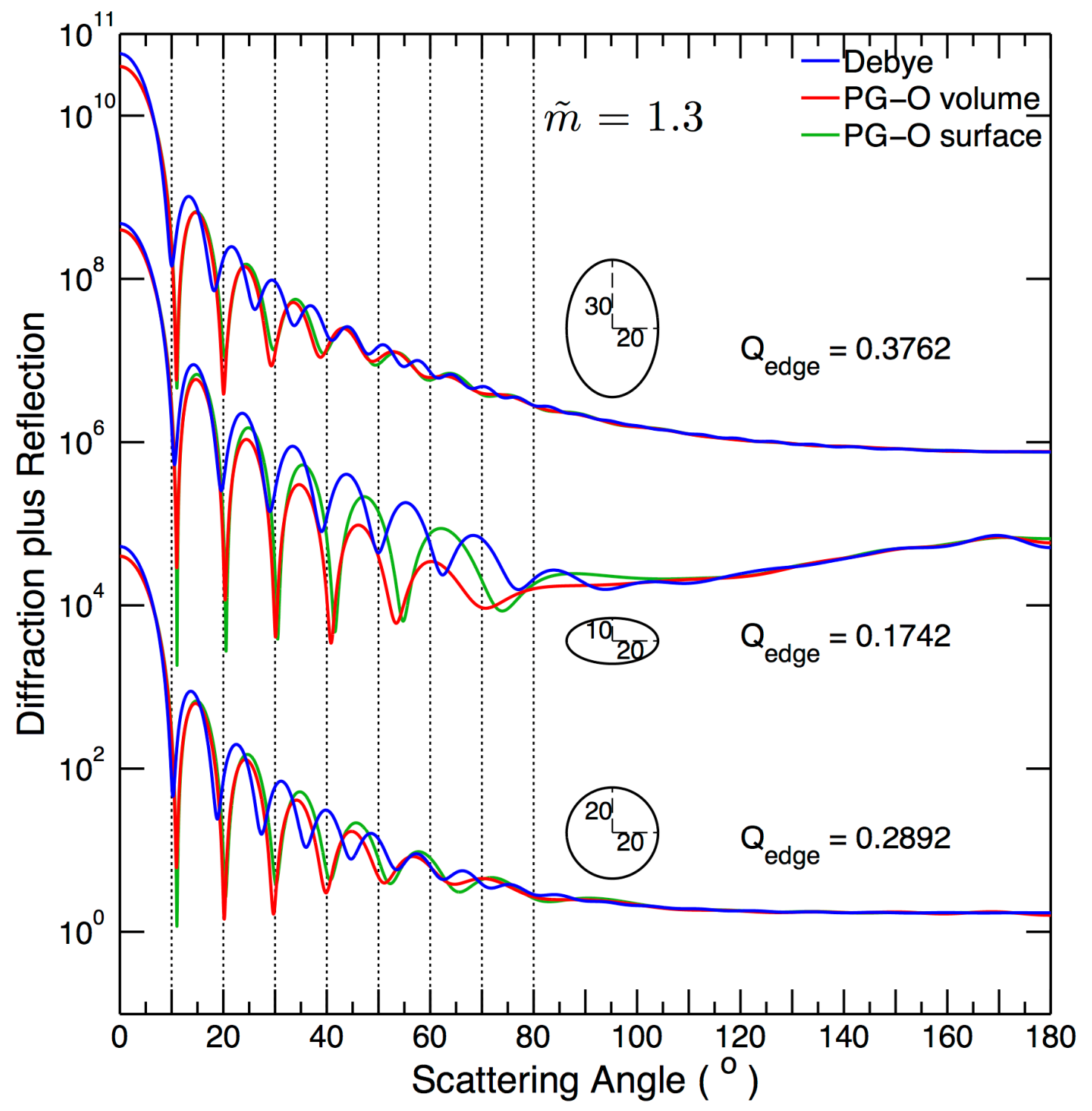

Fig. 2 Comparison of the phase functions of a sphere, an oblate spheroid, and a prolate spheroid computed from the Debye series and the PG-O approximation methods. For clarity, the phase functions associated with prolate and oblate spheroids are shifted upwards by factors of $10^{4}$ and $10^{6}$, respectively. 

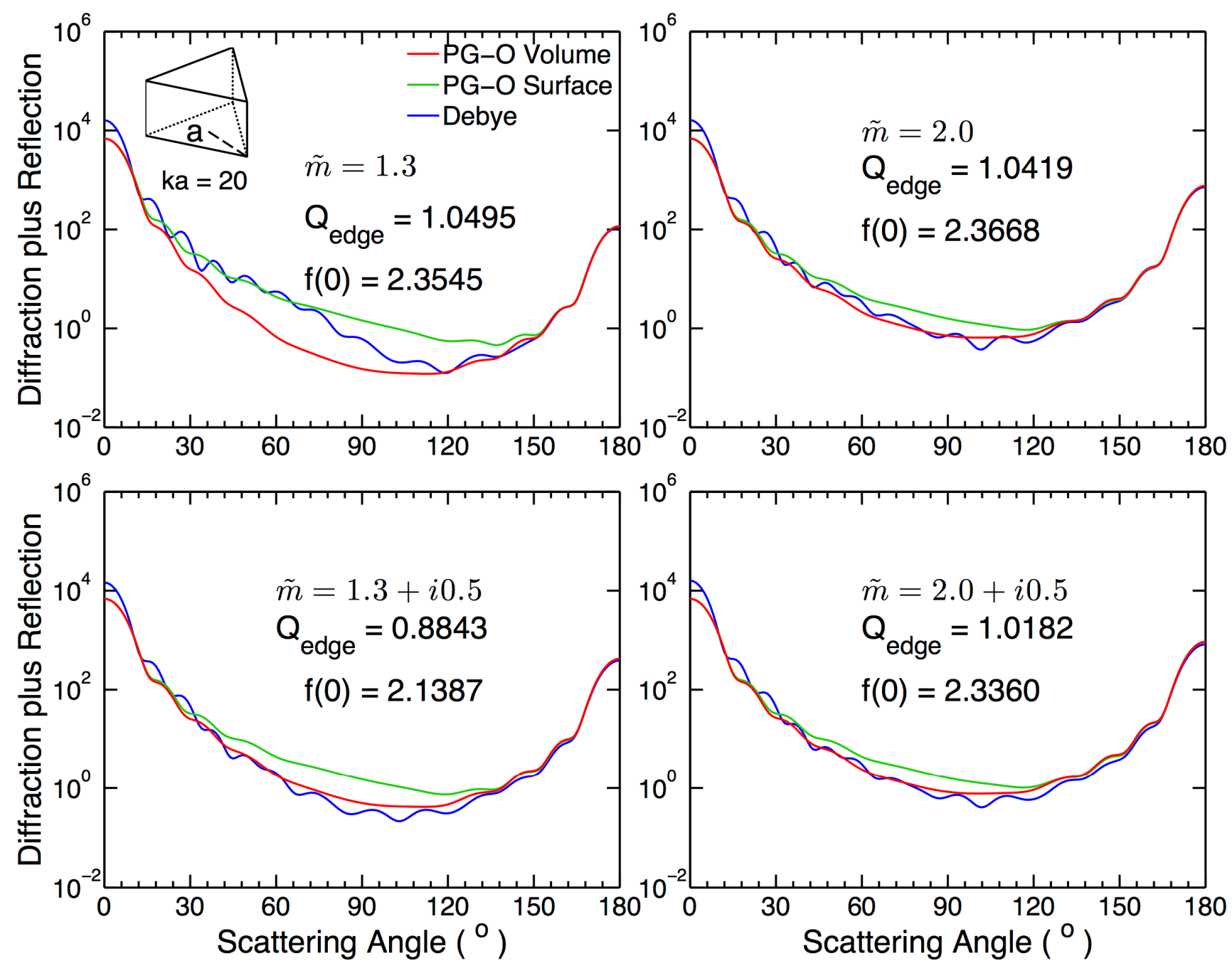

Fig. 3. Comparison of the phase functions of a triangular prism associated with diffraction plus reflection computed from the two PG-O approximation methods and the Debye series. 


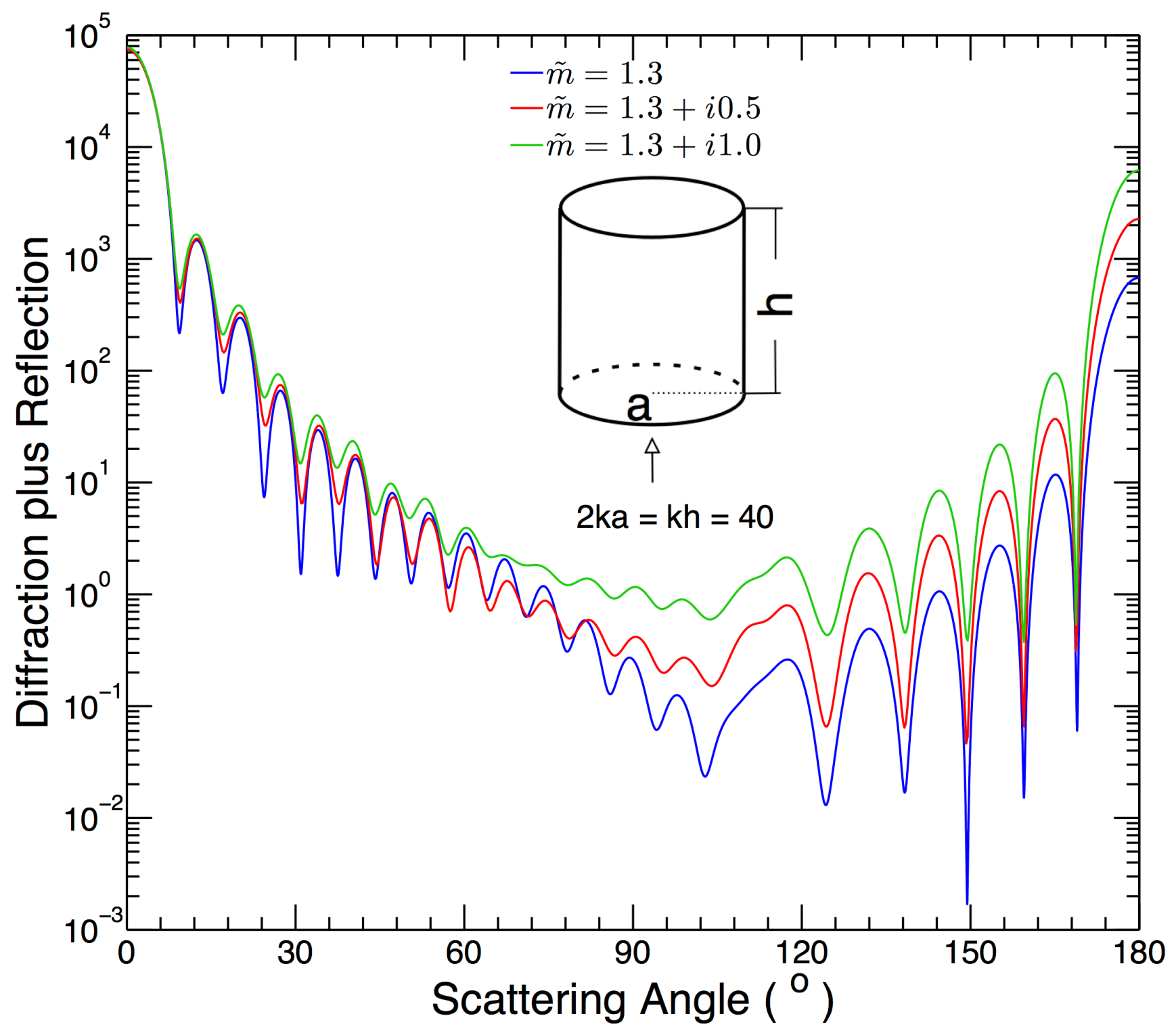

Fig. 4. The diffraction plus reflection pattern of a cylinder computed from the Debye series for three refractive indices $(1.3,1.3+i 0.5,1.3+i 1.0)$. The direction of the incident light is aligned with the symmetry axis. 

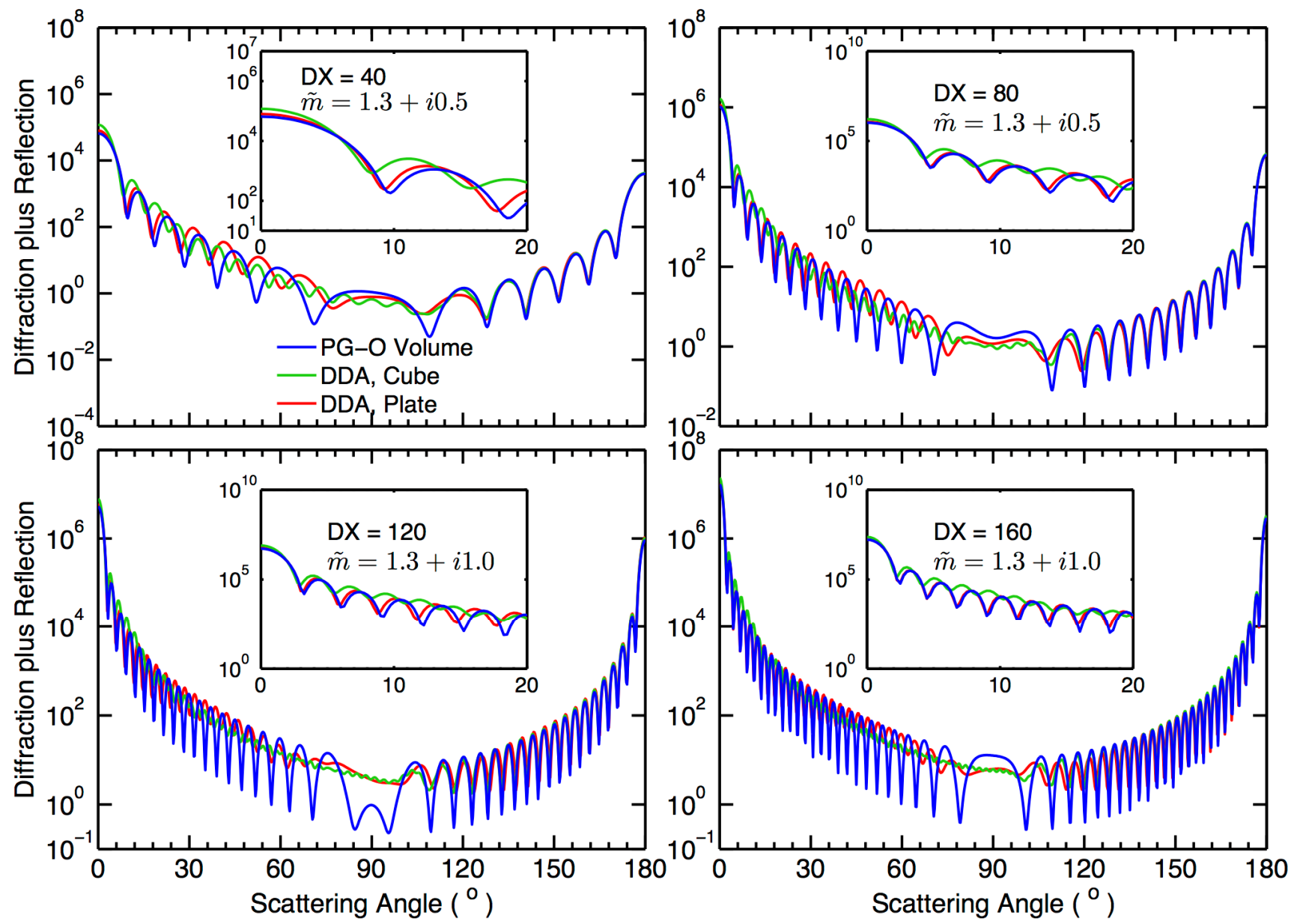

Fig. 5. The impacts of the edge effect on the diffraction patterns for a cube and a plate. The direction of the incident light is normal to the basal face (a square). DX is the edge length of the square. The plate aspect ratios (height/width) corresponding to $\mathrm{DX}=40,80,120$, and 160 are $0.2,0.1,0.1$, and 0.05 . 

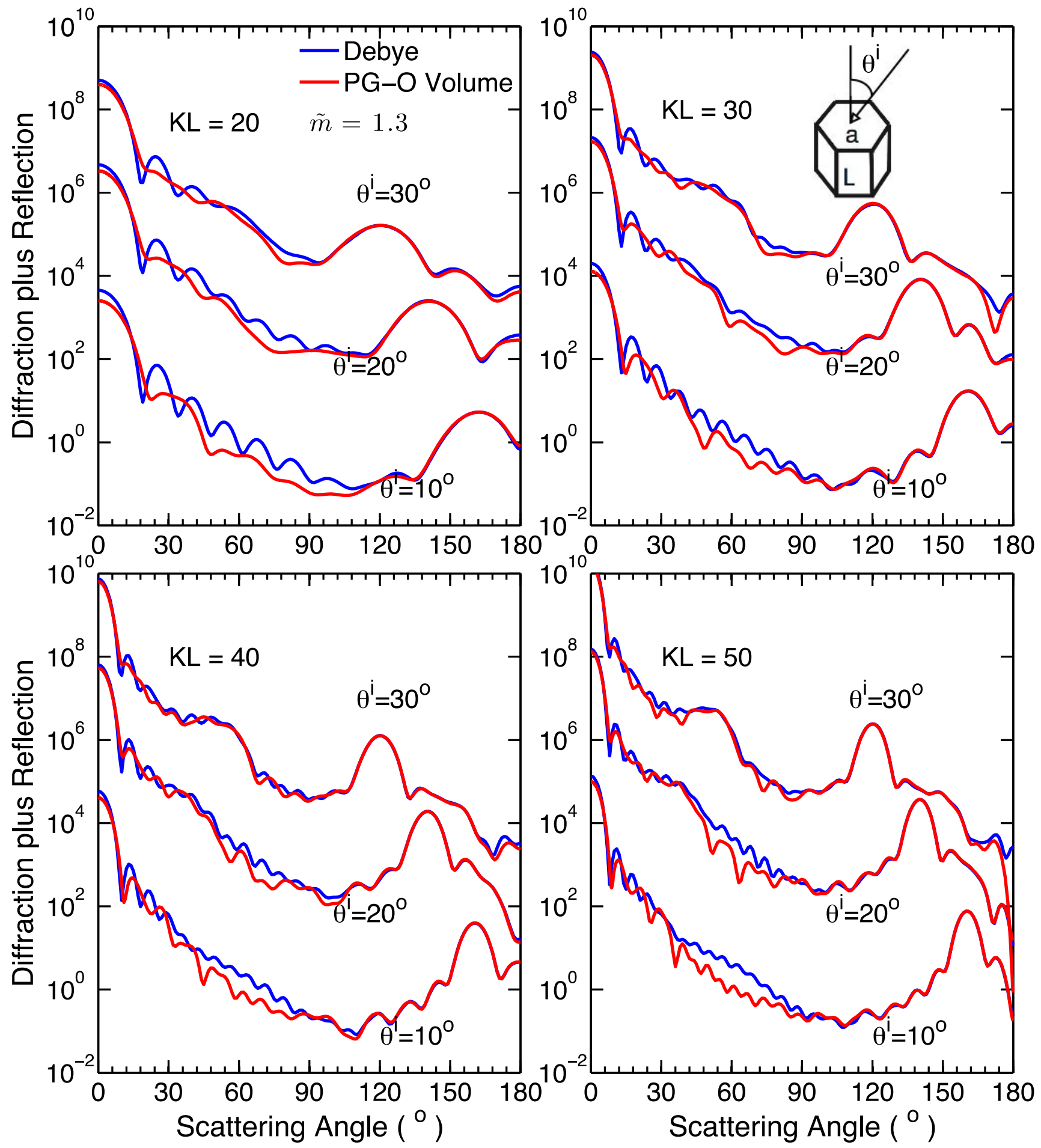

Fig. 6. Comparison of the phase functions for a hexagonal particle computed from the Debye series and the PG-O approximation method. The orientation of the particle with respect to the direction of the incident light is indicated with the angle $\theta^{i}$. The phase functions are averaged with respect to the scattering planes. 

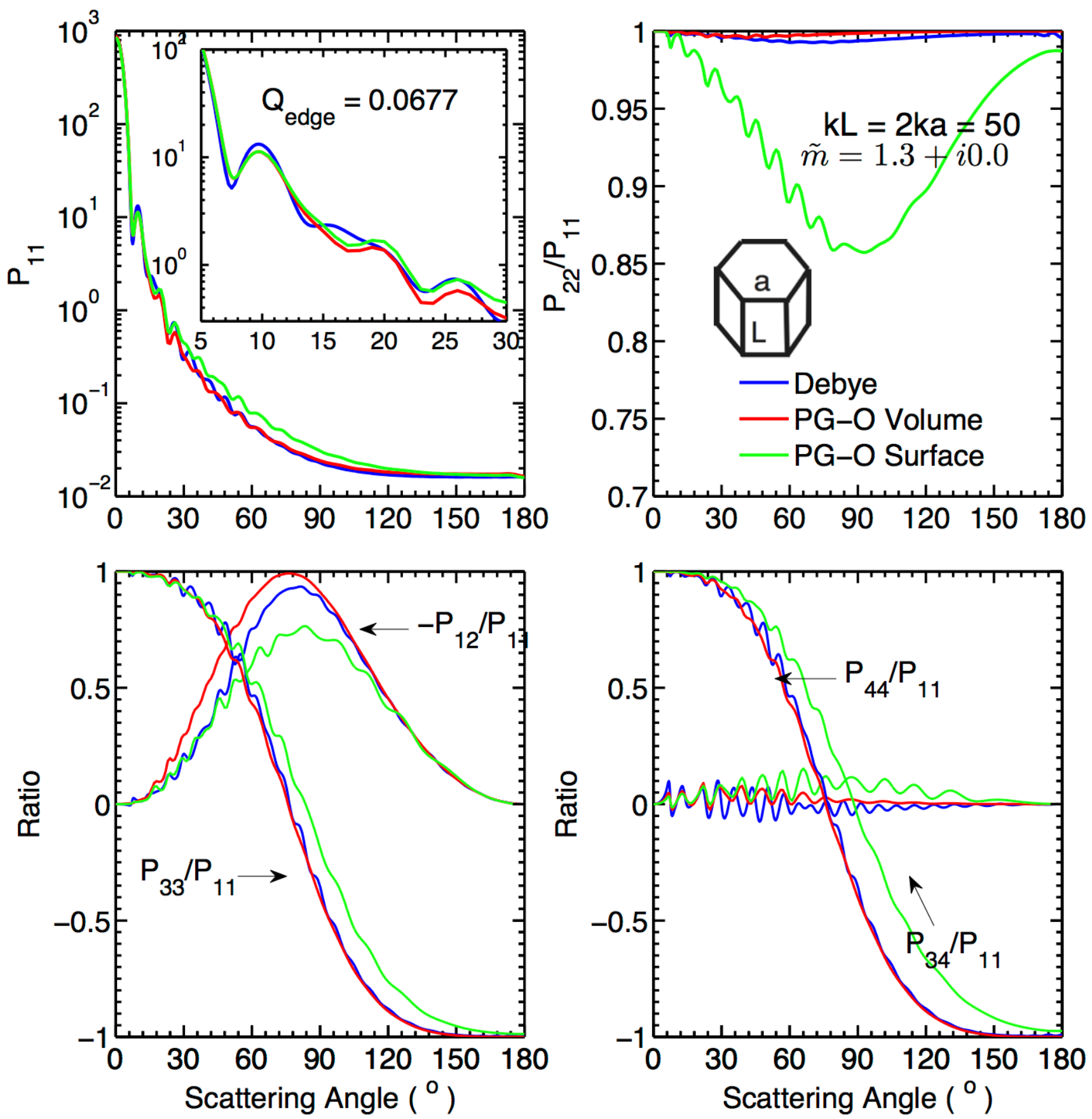

Fig. 7. Comparison of six phase matrix elements for a hexagonal particle associated with the diffraction and external reflection computed from the Debye series and the PG-O approximation method. In the bottom two panels, the $-\mathrm{P}_{12} / \mathrm{P}_{11}, \mathrm{P}_{33} / \mathrm{P}_{11}, \mathrm{P}_{34} / \mathrm{P}_{11}$ and $\mathrm{P}_{44} / \mathrm{P}_{11}$ ratios are shown with the same scales. 

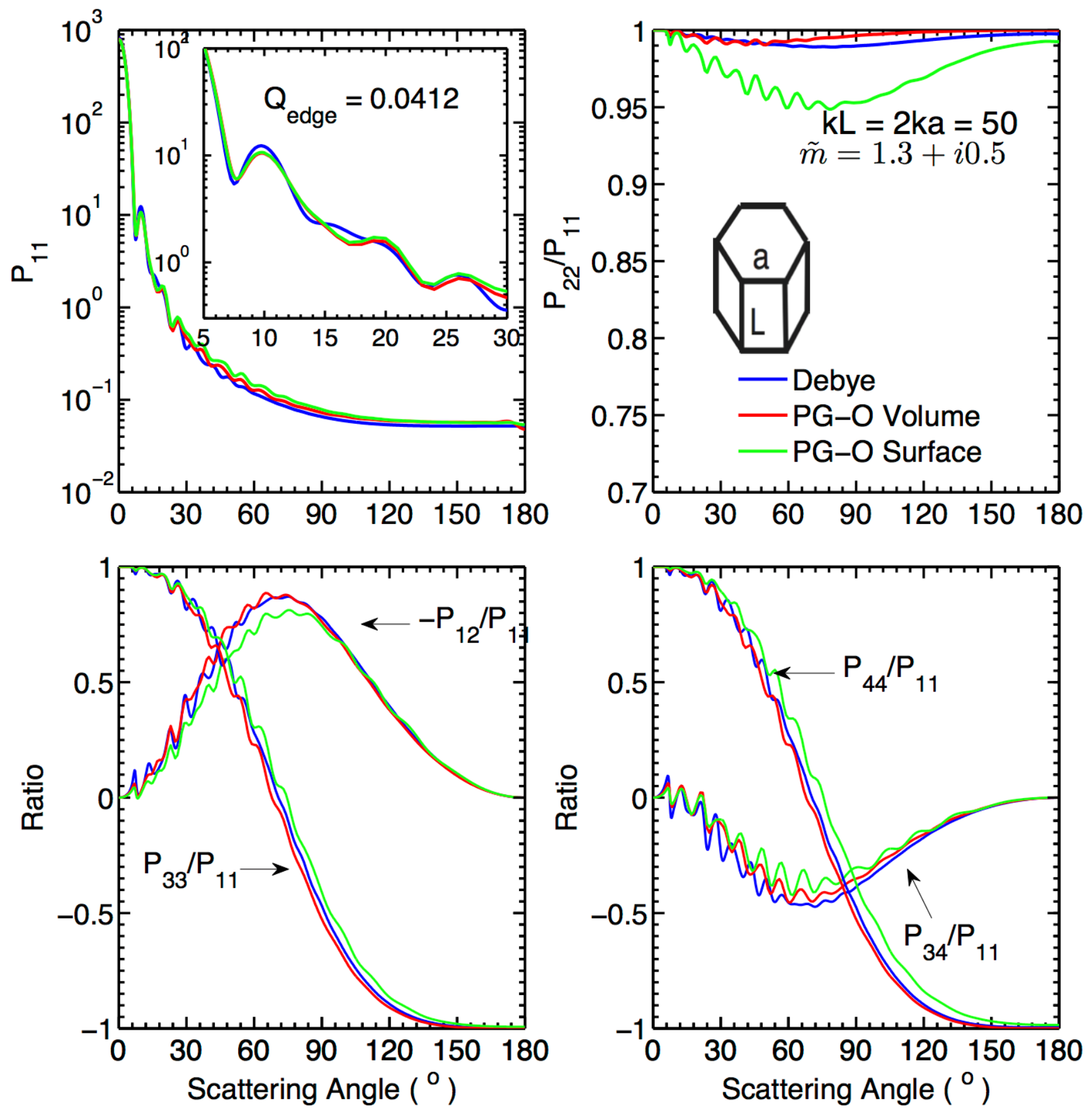

Fig. 8. Same as Fig. 7 except that the refractive index is $1.3+i 0.5$. 


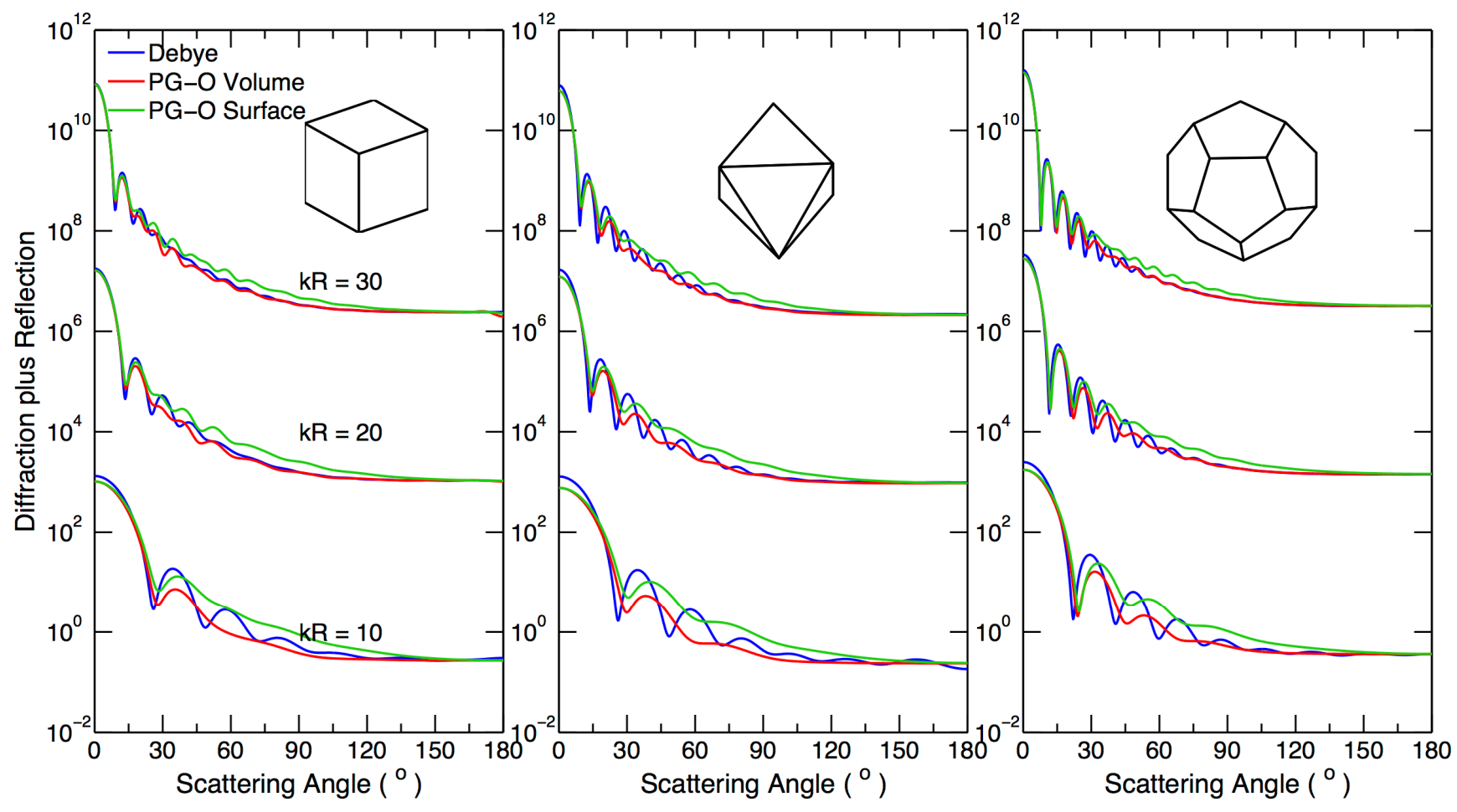

Fig. 9. Comparison of the phase functions for three faceted particles computed from the Debye series and the PG-O approximation method. $k R$ is the size parameter defined in terms of the radius of the circumscribed sphere. The refractive index is 1.3 . 